\title{
An Optical Instrument to Characterize Individual Dust Particles
}

\author{
Frank Giovane \\ NASA Headquarters, Washington, DC 20546
}

Bo A.S. Gustafson

Department of Astronomy, University of Florida, Gainesville, FL 32611

\author{
Philippe L. Lamy \\ Laboratoire d'Astronomie Spatiale, Les Trois Lucs, F-13012 Marseille, FR
}

\begin{abstract}
The conceptual design for an optical instrument capable of characterizing dust particles by in situ measurements is described. This instrument, the Integrated Dust Analyzer (IDA), measures the light scattered at several different angles as a particle passes through a pair of polarized light curtains. It is capable of defining or providing an indication of such characteristics as size, index of refraction, absorptivity, and aggregate structure.
\end{abstract}

\section{Introduction}

The characterization of cosmic dust by the examination of the light scattered by individual particles is a departure from the way dust observations have been made. These observations, by their remote nature, have by necessity depended on the integrated scattered light from many dust particles. Even in recent times such in situ spacecraft as Giotto have relied on integrated scattered light measurements to deduce the nature of the dust (Giovane, et al 1991). The recent development of low mass, high power laser diode arrays, combined with advances in photo detectors, have now made possible the development of a low mass high sensitivity instrument that can be practically carried by a deep space probe and which is capable of characterizing single dust particles by in situ measurement. This instrument, the Integrated Dust Analyzer (IDA), is described in the following paper.

IDA, by characterizing their size, index of refraction, absorptivity, and aggregate structure, would provide significant insight into the nature of particles encounted by a future space probe. This would be a powerful tool to better understand the origin and morphologic evolution of particles, and the properties of their parent body. On these space probes IDA could stand alone or be simply combined with other devices that can measure momentum, mass, velocity, direction, chemical composition, etc., so as to provide a broader understanding of the nature of the individual particles encountered. Variants of IDA can be produced to operate in a wide range of particle and spacecraft conditions. 


\section{Concept}

The concept of an instrument that can optically characterize individ ual dust particles is however not new. Various laboratory optical bench class instruments have been used in the past to make such scattering measurements, however, the characteristics of these labo ratory instruments differ significantly from those required of an instrument designed for space. Nevertheless, the fundamental principal of the laboratory and space instruments are the same. A particle enters the instrument through a particle entrance aperture, which may include baffles or stops to limit the angle at which the particles can enter. The particle then proceeds to a point where it is illuminated by a light beam, or light curtain, and there it scatters light into a group of photometers positioned at various angles around the scattering point. In some laboratory instruments it has been possible to trap the particle (by means of electrostatic field or by intense optical radiation) to allow a single photometer to be moved into different scattering angle positions while the particle is held in the light beam. The high relative motion of a particle relative to a typical spacecraft that might carry IDA precludes this latter approach.

The nature of the light curtain and the placement of the photometers in IDA was determined from a combination of theoretical study and practical considerations (e.g. volume, power, straylight). The result of this study calls for a system that utilizes six photometers positioned about the dust-light curtain intersection point. In this devict each photometer integrates light over a cone 6.42 degrees half angle centered at scattering an gles of 45,67.5, 90, 112.5, 135, and 157.5 degrees. The light curtains are composed of two linearly polarized light beams (whose electric vectors differ by 90 degrees). Each curtain is assumed to be $2 \mathrm{~mm}$ thick and capable of irradiating the particle with 50 watts $/ \mathrm{cm}^{2}$ at $635 \mathrm{~nm}$. Although other IDA applications might call for some modification to these parameters, the characteristics are sufficiently typical to illustrate the quality of information that can be obtained by IDA observations. These values were used in the simulations presented later in this paper.

\section{The Instrument}

The light source of choice for IDA is the laser diode array, which is capable of delive ing large amounts of radiant power in quasi monochromatic form, from a relatively low mass device with a small emitting area. A typical array is made up of several closely placed individual laser diode segments aligned in a grove cut in a material like Beryllium Oxide. The light from each laser diode segment can be collimated by a micro-lens. The light screen is rendered more uniform by modulating the density of a filter place on the light screen of the lenses. Approximately $10 \mathrm{w}$ of radiant power can be obtained per centimeter. Bars can be stacked for greater power.

Although the laser diodes are relatively efficient they are still heavy power users. One method of mitigating this power use is to activate the laser diodes only when a particle has entered the instrument. To accomplish this an alert screen is used. This screen is a low power light curtain with a single photometer used to detect the scattered light as a particles enters IDA. The power of this light curtain, because its purpose is detection rather than characterization, can be at least a 100 times less intense than the characterizing curtain. At an impact rate of 1 particle per second, the powe 
requirements for IDA's laser system when used with an alert screen can be very low (on the order of $100 \mathrm{mw}$ ). An optical system is used to condition the light from the array into a polarized, uniformly collimated curtain. Depending on the application, the light curtains can have a thickness of 1 to several $\mathrm{mm}$.

The collection of the scattered light from the particles can be achieved by the use a single lens and baffle system. However the use of a hollow cone-type collector (Winston, 1970) is preferred. The collector described by Winston is a non-imaging device, whose surfaces is in the shape of an off-axis rotated parabola. It is a more efficient concen trator of light than a simple truncated cone and can concentrate light better than the con ventional lens system. Although it is more complicated to construct, it has the ad vantage that it only accepts radiation emitted by the particle in a certain range of scattering angles about its position. This limited acceptance range of the collector restricts the light received from the particle in a way that is in effect independent of the distance the particle is from the collector. A narrow band interference type filter, centered on the laser diode wavelength, is used at the entrance aperture, where it assists in rejecting light from external sources that might enter through the IDA's dust entrance aperture.

The detector of choice for IDA is the Silicon PIN photodiode. It is reliable, and low in mass and power consumption. It also has a very large dynamic range and a very high quanturn efficiency, $76 \%$ at $980 \mathrm{~nm}$ and $65 \%$ at $630 \mathrm{~nm}$. Their NEPs are typically of about $1 \times 10 \mathrm{E}^{-14} \mathrm{~W} / \mathrm{Hz}^{1 / 2}$. In many space applications the detectors can be passively cooled to operate at about -40 degrees $C$ or less, greatly reducing the Silicon photodiode dark current.

The IDA processor and data requirements can be kept very simple. Although IDA can be operated without the use of a microprocessor, a simple microprocessor would allow signal selection and averaging to reduce the data requirements to about 400 bits/particle.

In most applications, the light from the laser diodes can be allowed to pass out of the IDA and into the outside environment where its dispersion will do no harm. The precise placement of stray light reduction baffles is however essential, and stray light suppression represents the most complicated aspect in building IDA.

\section{Simulation}

A simulation of the scattering as seen by the IDA photometers was carried out for spherical particles between 0.1 and 100 micron diameter traveling at $100 \mathrm{~m} / \mathrm{s}$ relative to IDA. These included water ice, Astronomical Silicate (Draine and Lee, 1984), an organic refractory (Greenberg and Hage, 1990), and metals. The number of photons received by the photometers are sufficient to detect particles as small as 1 micron. The linear dimension of a particle can be gauged from the amount of light it scatters into the photometers. A rough estimate can be obtained simply by summing the scattered light from the two perpendicularly polarized light screens. This method of size determination appears to be insensitive to the material of the particle. 
Metallic particles can also be separated from dielectrics based by the absence of the 90 degrees minimum in one of the polarization components. The minimum does not develop in the scattering from metallic particles until they are too small to scatter a detectable signal.

The procedure for determining the refractive index involves first determining the imaginary part of the refractive index from the degree of linear polarization at the smallest scattering angles. This is simplified by knowledge of the approximate particle size from the magnitude of the scattered light from the perpendicularly polarized beam. The real part of the index is determined from the variation of the degree of polarization with scattering angle. The refractive index is highly indicative of the material type, although a specific material can not be uniquely assigned to the particles based on the refractive index alone. However, the refractive index defines a material's optical properties and the particles so classified. In association with other measurement devices, such as a mass monitor, an estimate of the density will assist the determination of the material type. Although the run of the degree of polarization is also highly diagnostic, care must be taken to avoid misinterpretation.

Compact particles can be distinguished from porous aggregate structures, however there are spherical particles with comparable polarization and a similar angular dependency of the scattered intensity as that of some aggregates. The magnitude in scaltering generally is usually dramatic, so that misclassification can be avoided and the existence of ambiguous cases can be anticipated. Equal mass solid particles and aggregates will not create similar scattering. The simulation considered equal cross section spheres where the materials have been evenly distributed. The scattering magnitudes in perpendicularly polarized light is noticeably flatter for the aggregates than for any of the approximations and the polarization differ.

\section{Conclusion}

The Integrated Dust Analyzer, IDA, is a powerful tool for characterizing individual dust particles, and for providing information that has hitherto not been available from space. Advances in the technology of laser diode arrays and detectors makes it possible to construct an instrument that can be highly reliable and frugal in its need of spacecraft resources. Variants of the design can be developed to accommodate a range of requirements and conditions that may be experienced by future space missions.

\section{References}

Draine, B.T., \& Lee H.M. 1984, Ap.J. 258, 89-108

Giovane, F, et al. 1991, Appl. Opt. 30, 2579

Greenberg, J...1., \& Hage, J.I. 1990, Ap.J. , 361, pp 260-274

Winston, R. 1970, JOS i I, 60, 245-7 